\title{
Microdissection of chromosome 2 - between-arm intrachromosomal insertion
}

\author{
Ursula Friedrich ${ }^{1}$, Mette Houman ${ }^{1}$, Jette Sandgaard ${ }^{2}$, Anni Rosgaard ${ }^{3}$ and Lone Sunde ${ }^{2}$ \\ ${ }^{1}$ Microdissection Department, Institute of Human Genetics, University of Aarhus; ${ }^{2}$ Department of Clinical Genetics, \\ Aarhus University Hospital; ${ }^{3}$ D epartment of Obstetrics and Gynaecology, Central Hospital, Holstebro, Denmark
}

This report describes a mother with a balanced intrachromosomal insertion of band q22 on chromosome number 2 into band p24 on the same chromosome. She had had four spontaneous abortions and two induced abortions. One foetus had a suspected obstruction of the uretero-pelvic part of the urinary tract and monosomy of band $2 q 22$, the other foetus had anencephaly and trisomy of band $2 q 22$. By microdissection we have generated a painting probe from the mother's abnormal short arm of chromosome 2 (der2p probe). This family specific probe will be used in future pregnancies for precise diagnosis. European Journal of Human Genetics (2000) 8, 393-395.

Keywords: microdissection; intrachromosomal insertion; chromosome region (2)(q21q23); agangliosis

\section{Introduction}

Few cases of intrachromosomal insertions have been published so far (for review see Madan and $\mathrm{Menko}^{1}$ ). An insertion arises due to a three-break rearrangement. A chromosome segment is shifted from one arm into the other. Three-break rearrangements are about ten times less frequent than twobreak events. The estimated frequency is 1:5000 live borns (Chudley et $\mathrm{al}^{2}$ ). Insertions are difficult to detect. They are often mistaken for inversions or duplications. A single crossover between any of the three breakpoints leads to unbalanced recombinants either with duplications or deletions of the inserted segment. For an insertion carrier the risk of an unbalanced progeny is at least $15 \%$ and may reach $50 \%$ for particular insertions.

We present a family with an abnormal chromosome 2 . The generation of arm-specific probes and a family-specific probe by microdissection of the abnormal short arm of chromosome 2 helped us in the precise diagnosis. The probe will be of value in future pregnancies.

\author{
Case history \\ The proband, foetus II-4 (Figure1), was the product of a \\ pregnancy terminated by induced abortion in week 12 \\ Correspondence: Ursula Friedrich, Department of Microdissection, \\ Institute of Human Genetics, The Bartholin Building, University of \\ Aarhus, DK-8000 Aarhus C, Denmark. Tel +458942 1684; Fax: \\ +458612 31 73; E-mail: zitta@humgen.au.dk \\ Received 14 June 1999; revised 5 November 1999; accepted \\ 11 November 1999
}

because of anencephaly. No other malformations were observed. Routine chromosome examination in QFQ-banding revealed an abnormal short arm of one chromosome no. 2. Chromosome examination of both parents suggested the same abnormal chromosome no. 2 in the 33-year-old mother. She was physically and psychologically normal.

One year earlier, she had another induced abortion in week 22. The foetus, II-3, had hydronephrosis and multicystic kidneys. An obstruction of the uretero-pelvic part of the urinary tract was suspected. No other malformations were described. Routine chromosome examination in QFQbanding in this foetus had shown normal results.

Before these two induced abortions, the mother had had two spontaneous abortions in week 14 (II-1) and week 10 (II-2) in a previous marriage. Subsequently she again aborted spontaneously a twin pregnancy in week 10/11 (II-5 and II-6).

\section{Method}

Microdissection probes were made from the short and long arms of a normal chromosome 2 as well as the abnormal short arm of the derivative chromosome 2 from the mother. The microdissection protocol used is a combination and a modification of three methods described previously (Senger et $\mathrm{al}^{3}$; Müller-Navia et $\mathrm{al}^{,}{ }^{4}$ Engelen et $\left.\mathrm{al}{ }^{5}\right)$. Two important steps should be mentioned.

A preamplification step was performed. The collected material in the collection drop was cycled for 15 cycles at 
$30^{\circ} \mathrm{C}$ and $50^{\circ} \mathrm{C}$ for $1 \mathrm{~min}$ each in a Hybaid sprint PCR machine with a heated lid. By this step DNA in the further amplification seems to be more accessible for primers and polymerase. For amplification we used the following modified DOP primer (5' - CCG ACT GCA G N ${ }_{6}$ ATG TGG - 3').

Fluorescence in situ hybridisation (FISH) was performed following the usual chromosome in situ suppression (CISS) protocol.

\section{Results}

The pedigree and the results from QFQ-banding and the different hybridisations are shown in Figure 1. Hybridisation with the short arm probe of a normal chromosome 2 to the mother's chromosomes (I-2) showed signals only on the short arms of both chromosomes no. 2. Hybridisation with

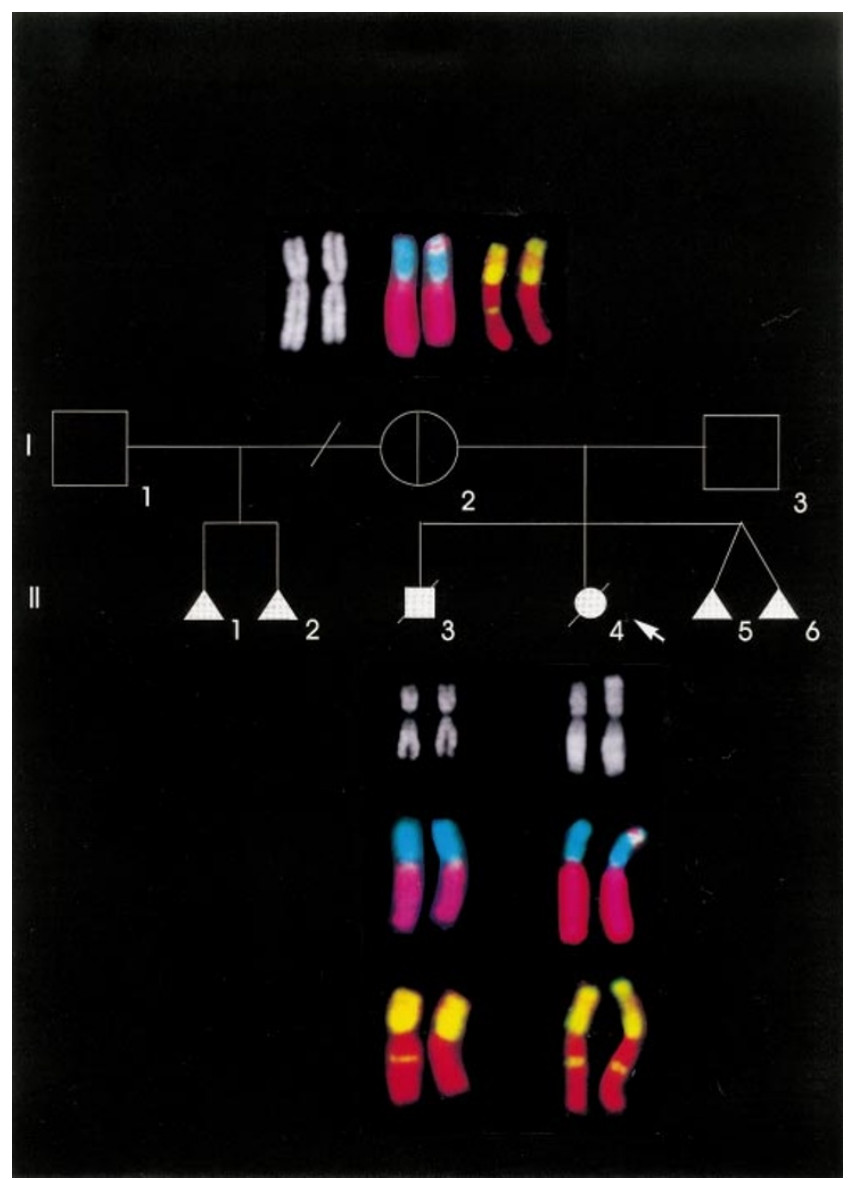

Figure 1 Pedigree of the family. The chromosomes are shown in QFQ-banding, in painting with an arm specific probe from the long arm of chromosome 2 ( $p$ Dapi blue; qred) and in painting with the der $2 p$ probe from the mother (yellow). FISH was performed on chromosomes of the affected individuals, the abnormal chromosome 2 always being placed to the right. $\mathrm{I}-2$ is a carrier of a balanced intrachromosomal insertion, II-3 is monosomic and II-4 is trisomic for the inserted segment of the long arm. the long arm probe showed signals on the long arms of both chromosomes, and on the short arm of one chromosome no. 2 a small signal was seen at band $2 p 24$. Thereafter, we used the specific der ( $2 p)$ probe from the mother and painted metaphases of the mother. Both short arms of no. 2 were painted with this probealong with band q22 on the long arm of the normal chromosome no.2. This showed that the mother had an insertion of band q22 into band p24 of chromosome no.2. Her karyotype is $46, X X$, ins(2)(p24q21q23). The orientation of the small, inserted segment towards the centromere is not clear.

Foetus II-4 had an abnormal chromosome no. 2 in QFQbanding. Hybridisation with the short-arm and long-arm probes of chromosome no. 2 showed the same insertion of band q22 in p24 as in the mother. Moreover, hybridisation with the maternal der( $2 p)$ probe revealed the band $q 22$ was also present in both long arms. Thisfoetus is thus trisomic for band $2 q 22$. The karyotype is $46, X X, \operatorname{rec}(2)$ dup(2)(q21q23) ins(2)(p24q21q23)mat.

Foetus II-3 presumably had two normal chromosomes no. 2 in QFQ-banding. There was no insertion as indicated by hybridisation with the probes from the short and long arm of chromosome no. 2. Hybridisation with the maternal der(2p) probe revealed only one band q22 in the long arm of one chromosome no. 2. This foetus is thus monosomic for band 2q22. The karyotype is $46, X Y$, $\operatorname{rec}(2) \operatorname{del}(2)(q 21 q 23)$ ins(2)(p24q21q23)mat.

\section{Discussion}

The intrachromosomal insertion in the mother could be disclosed by painting with a long arm specific probe from no. 2. Hybridisation with the der( $2 p)$ specific probe from the mother showed a clear signal which was localised to band q22 of the mother's normal chromosome 2. With this family specific maternal der(2p) probe it was not only possible to identify a balanced insertion in the mother but also to determine a partial trisomy of band $2 q 22$ in one foetus (II-4) and a partial monosomy of band $2 q 22$ in the other (II-3). The foetus with deletion (2)(q21q23) had ureter and kidney dilations which were suspected to be due to an uretero-pelvic obstruction. In a recent report about a new contiguous gene syndrome with Hirschsprung disease, microcephaly, mental retardation and distinctive facial phenotype, two of the six patients had dilated renal calyces and ureter. One of these two patients had a deletion of the same chromosomal region (M owat et $\mathrm{al}^{6}$ ). Another infant with deletion (2)(q21q23) had aortic stenosis in hypoplastic right heart syndrome but also cryptorchidism and mild hydronephrosis (Sharma et $\mathrm{al}^{7}$ ). The present foetus with a deletion of 2 q22 was aborted in the 22nd week of pregnancy. The diagnosis of Hirschsprung disease was not made. But since agangliosis of both bowel and ureters has been seen previously (Bamforth et $\mathrm{al}^{8}$ ), it cannot be excluded that the ureter dilations, dilated renal calyces and hydronephrosis were caused by agangliosis in the 
organs involved, as in the agangliosis in Hirschsprungs disease, and thus were caused by disturbance of the same gene localised at $2 q 22$.

In carriers of intrachromosomal insertions, the risk of unbalanced offspring is rather high. Madan and $\mathrm{Menko}^{1}$ obtained a risk figure for unbalanced progeny of $15 \%$. For particular insertions they suspect a risk close to the maximum theoretical risk of $50 \%$. The present family with six abortions may belong to this group.

Prenatal diagnosis should be offered to such families. In the present case, microdissection and utilisation of the specifically tailored probe of the maternal derivative chromosome no. 2 will enable us to obtain a reliable diagnosis in future pregnancies.

\section{Acknowledgements}

DrClaus Lund is thanked for the foetopathological examination of two foetuses. The study was financially supported by the Danish Research Council, grant no. 9802737 and the Rosalie Petersens Fond, grant no. $62036 / 3$.

\section{References}

1 Madan K, Menko FH: Intrachromosomal insertions: a case report and a review. Hum Genet 1992; 89: 1-9.

2 Chudley AE, Bauder F, Ray M, MCAlpine PJ, Pena SDJ, Hamerton JL: Familial mental retardation in a family with an inherited chromosome rearrangement. J Med Genet 1974; 11: 353-363.

3 Senger G, Lüdecke H-J, Horsthemke B, Claussen U: Microdissection of banded human chromosomes. Hum Genet 1990; 84: 507-511.

4 Müller-Navia J, Nebel A, Oehler D, Theile U, Zabel B, Schleiermacher E: Microdissection and DOP-PCR-based reverse chromosome painting as a fast and reliable strategy in the analysis of various structural chromosome abnormalities. Prenat Diagn 1996; 16: $915-922$.

5 Engelen JJM, Albrechts JCM, Hamers GJH, Geraedts JPM: A simple and efficient method for microdissection and microFISH. J Med Genet 1998; 35: 265-268.

6 Mowat DR, Croaker GDH, Cass DT et al: Hirschsprung disease, microcephaly, mental retardation, and characteristic facial features: delineation of a new syndrome and identification of a locus at chromosome 2q22-q23. J Med Genet 1998; 35: 617-623.

7 Sharma J, Friedman D, Schiller M, Flynn P, Alonso ML: Aortic stenosis in hypoplastic right heart syndrome, associated with interstitial deletion of chromosome 2. Int J Cardiology 1997; 62: 199-202.

8 Bamforth JS, Leonard CO, Chodirker BN et al: Congenital diaphragmatic hernia, coarse facies and acral hypoplasia: Fryns syndrome. Am J Med Genet 1989; 32: 93-99. 